\title{
Material and design challenges in phase change memory
}

\author{
Wenfa $\mathrm{Ng}$ \\ Department of Chemical and Biomolecular Engineering, National University of Singapore, \\ Email: ngwenfa771@hotmail.com \\ ORCID ID: https://orcid.org/0000-0001-7629-2140
}

\begin{abstract}
Together with display and input, storage and processing are requisite to computing. While storage technologies have improved tremendously in capacity and speed over the years, the basic principles enabling information storage into digital 1 and 0 remains the same: induction of phase change in the storage substrate. But recently, there has been much research into structural phase change material (SPCM) and exploration of its possible use in various types of memory storage applications. Despite unconventional use of structural change between amorphous and crystalline state as well as that between crystal structures for encoding information, key barriers for its widespread use remains access speed, capacity to cost ratio, and fidelity of storage. Hence, given the performance requirement of SPCM for memory applications, what are the material and design considerations that feed into translating a promising application into a practical reality? Given the important role of kinetic and thermal energy in structural organization of a phase change material, precise characterization of structural change in the material with external physical factors such as heat, voltage and current, is critical for storage material design. Next comes the precision at which individual memory cells for storing single bits of information could be defined reproducibly and at high fidelity using SPCM. In congruent with memory cell definition lies the equally important aspect of constraining the field characteristics used in modulating the phase state of the memory medium. Specifically, while heat is useful for mediating the "melting" of a crystalline material into its amorphous state, heat conduction is less useful for transferring the "switch command" from the effector to the memory material. More importantly, choice of structural phase change material for memory applications likely revolves around those where individual memory cells could be defined in a cross array format, which is amenable to high density information storage. Durability and fidelity of information storage are additional factors of design that favours selection of SPCM with phase change occurring at narrow operating windows without hysteresis over extended cycling. But, the most important requirement is speed of access. To this end, energetic cost of phase transitions might affect operation of the phase change memory at the system level: for example, usage of large current for high energy transition step may impact on device durability. Ultimately, there is a fundamental limit on the number of reproducible phase transitions in a SPCM before fidelity of information storage is no longer guaranteed. Hence, what are the drivers for uptake of phase change memories in consumer devices? Performance gains must be realized in access speed, storage capacity, form factor, and fidelity of information storage for practical application of structural phase change memories.
\end{abstract}


Keywords: phase change material, amorphous, crystalline, operating window, speed of access, fidelity of storage, energetic cost,

Subject areas: material science and engineering, physics, chemistry,

\section{Introduction}

Phase change memory is a relatively new concept in memory applications. ${ }^{1-4}$ It is coveted for its nonvolatile nature, where a permanent record of data is stored in memory even when the computer is switched off. However, the concept itself presents significant challenges to material science and memory design given the incompatibility between properties of most materials and the performance requirements of computing (i.e., low latency, fast read/write operations, high number of cycles without fading, accurate reproduction of stored data, reversibility etc.). ${ }^{5}$ For memory applications, the material chosen must exhibit different "states" or properties when subjected to different conditions. In conventional memory, this comes about through magnetization and demagnetization of a ferromagnetic material that serves as the storage medium for information, encoded in digital $0 \mathrm{~s}$ and $1 \mathrm{~s}$. This method of information encoding presents advantages of reversibility, fast memory retrieval, and relative low cost at high volume of production. This set the design parameters for phase change memory to emulate.

\section{Discussion}

\subsection{Computer memory applications require dimensionally limited phase change material}

Phase change memory uses change of phase (e.g., between amorphous and crystalline states or between different crystal structures), to encode and store information. ${ }^{3,6,7}$ However, given the disorganized and uncontrolled nature of phase transformation from crystalline to amorphous states, attempts in encoding information in the crystalline states is vulnerable to the problem of amount of useable memory recoverable from the material after an erasure process of changing the memory into the amorphous state. More importantly, modern memory applications require high density storage for the creation of storage medium at low cost to the consumer. Hence, to pass muster as an information storage medium, phase change material's transitions from crystalline to amorphous states must be dimensionally limited. ${ }^{3,8}$ Specifically, initiation of transition by external factors such as the Joule heating from a brief but strong current must not lead to progressive change of phase in large areas of the storage medium. ${ }^{7}$ From another perspective, memory address on the phase change memory must be individually addressable by the mode for induction of phase change, where exertion of a physical force would only enable reading and writing operations to be performed at specific locale in the memory. Phase change materials vulnerable to large area change of phase (whether the memory elements are slated for read or write operations) are thus not suitable for memory storage applications. 


\subsection{Requirement of fast and reproducible phase change in computer memory applications}

Computers are tools that help process data at speeds faster than what humans are capable of. Hence, memory read/write operations must be fast and accurate. ${ }^{7}$ However, depending on the types and extent of phase change involved, phase transitions may not be suitable for memory storage given the relative slow speed of physical phase change such as from amorphous state to crystalline state, compared to conventional magnetization induced information storage, which is fast, accurate and reliable over multiple read/write operations. This is one area where phase change material design and engineering must innovate to afford a material suitable for memory applications, particularly the very fast random access memory.

Physical constraints dictate that phase change is by default slower than magnetization; thus, how should materials engineers and scientists devise a memory platform out of phase change material with such intrinsic slow kinetics of operations. One possible solution necessitates the creation of physically isolated memory cells, where each memory location is small compared to the overall size of the memory unit. Consider the case of a memory cell, transition from amorphous to crystalline state due to induction by a strong brief pulse of current, encodes a $1 \mathrm{~s}$, which, in turn, could be reverted by another current that "melts" the crystalline state and returns the amorphous state. The question necessarily arise, is the second amorphous state similar to the initial one? Or operationally, would the same current help convert the second amorphous state back to a crystalline one? Hence, concept of hysteresis of materials must be considered during materials development and selection of the final composition of the phase change material for memory applications. Specifically, for use as memory, hysteresis is not desirable given the need for the material to exhibit consistent behaviour across the same set of physical transitions and operating conditions; i.e., for phase change memory, the material must convert between amorphous and crystalline states at the same transition voltage and current density. Is this achievable over a long run, which is necessary for commercial relatively low-cost memory modules common in consumer laptops and mobile devices?

The question posed above is still being actively pursued in research labs around the world, both commercial and academic. Specifically, there is likely to be a fixed number of cycles between amorphous and crystalline states before there would be material degradation or loss of performance in reliability of encoding information. If the number of reliable transitions between states is low, the cost of phase change memory may not be commercially viable as a memory module for low cost computing devices. In the same vein, a material with relatively low reliability in memory storage would also not find use in high end computing applications. 


\subsection{Lateral scaling to high storage density is necessary for commercial application of phase change memory}

High density of storage has always been the desired goal of memory manufacturers given the need for high volume of storage in a low weight form factor. Thus, phase change memory could not buck this trend and must be amenable to lateral scaling where high density of memory cells could be created in a substrate in a reproducible and high resolution manner. ${ }^{7}$ But, transition from crystalline to amorphous phase suggests that the desire for a small memory cell capable of reversible storage of information through change of phase may not be as readily realizable compared to magnetization. Specifically, amorphous state of material is inherently disorganized and, more importantly, transition from crystalline to amorphous phase may not yield to easy control given the tendency of the amorphous state to "spread" to neighbouring molecules in a crystal structure. Thus, material design and crystal engineering are needed to create "demarcation landmarks" at the molecular level to define the physical size of the memory cells, and more importantly, prevent a cascade amorphous transition in the material when phase change was induced. Doing so would help generate a substrate platform where work could be effected to realize a fast, high capacity, reliable, highly reproducible phase change memory storage medium.

\subsection{Methods for inducing phase change must be precise, fast and reversible}

The method by which phase transition could be exerted in the phase change memory is another area requiring much materials discovery, and subsequently, optimization at the proof-ofconcept stage. Generally, the mode to induce phase transition in materials for memory storage must be precise and able to address individual memory units, not resulting in permanent loss of phase transition capability (i.e., not pushing the material beyond the bounds of the phase diagram), able to effect phase change quickly, and, most importantly, would not cause irreversible damage to the memory module. By and large, type of phase change material and its mode of induction are intricately intertwined, given that the properties of the material constrain the physical method for inducing phase change. For example, the relatively recent 3D Xpoint fast phase change memory material jointly developed by Intel and Micron requires short, strong current for inducing amorphous transition from the crystalline state through a Joule heating effect. While heating effect could be provided by direct heat, the relatively slow nature of heat conduction meant that Joule heating effect should be induced through the provision of a direct current to individual memory cell, which meant that a layer of heating element is needed for each layer of memory cells. Such a design meant that phase change memory may remain relatively expensive in the foreseeable future due to complexity in its engineering. Recent research has helped ameliorated the problem by demonstrating the use of smaller current and better thermal insulation dielectrics surrounding the memory cell to afford faster memory switching and response. ${ }^{9}$ 


\subsection{Key performance metrics for phase change memory}

Thus, phase change material for memory applications must fulfil the following performance requirements: (i) reliable storage of information in crystalline state, (ii) fast and reproducible transition between amorphous and crystalline states or between crystal structures, (iii) high density storage with each memory cell individually addressable by phase change agent, (iv) transition to amorphous state definable by physical constraints natural or built into memory module, (v) high number of phase change transitions without degradation in memory storage function, (vi) amenable to control by phase change agent during phase transition with preference for field based phase transition agent such as heat, and (vii) able to be fabricated in a manner allowing definition of small memory cells repeated across the entire memory module, for example, a cross point array structure such as in 3D Xpoint memory. Thus, as material choice defines future mode of operation and performance, there has been extensive research into material synthesis for phase change memory. However, commercial and practical application asks a fundamental question: what are the speed, capacity and cost advantage of phase change material compared to conventional memory materials? More importantly, given the fairly exacting performance demands, is individually addressable memory cell at small micron scale realizable?

\subsection{Liquid crystal display offer guidance for the design of phase change memory}

To this end, the advent of liquid crystal displays, and more modern high definition display technologies evolving from the concept of building an entire display with pixel as building blocks is a case in point. Similar to the concepts necessary for realizing phase change memories, liquid crystal display exhibit two distinct and definable states of matter: amorphous and crystalline. Most importantly, the two phases are interchangeable after induction by an externally applied phase change agent. Refinement of material construction further enabled the definition of a pixel where the properties of the liquid crystal could be modulated independently of a neighboring group of liquid crystal molecules belonging to another pixel. Such defined modulation of single or groups of liquid crystals calls for a phase change agent with precision targeting capability able to address specific groups of crystals. Identical to the concept underlying 3D Xpoint phase change memory, liquid crystal displays are also arranged in a cross point array at the molecular level; thereby, allowing individual groups of liquid crystal molecules (i.e., a pixel) to be defined and addressed by the phase change agent in a way similar to finding a point on a square grid. Thus, by controlling the phase in each pixel, groups of pixels could encode information present in the original image that the entire liquid crystal display is supposed to represent. Similarly, phase change memories could utilize the knowledge learnt in liquid crystals display technologies to encode information through a series of concatenated 1 and 0 at the molecular level, in a grid like fashion.

\section{Conclusions}

Collectively, phase change materials could only supplant current best-in-class memory technologies such as the hard disk drives if it offers enhanced performance characteristics such as 
faster read/write speed, high storage capacity at low cost, high fidelity of durable memory storage, and the ability to withstand the rigors of daily use by consumers. In particular, speed of phase transition and the ability to create individual memory cells whose phase change (and thus, memory state) could be reversed with a phase change agent with spatial precision down to a sub memory cell level are key attributes of a candidate phase change material that could proceed to subsequent rounds of engineering and optimization. But, what does the future portend for this emerging class of memories? Phase change memories may afford a memory platform whose read/write speeds could be further developed and memory capacity and durability enhanced. But, what is the reason for a switch towards phase change memory? Current trends in adoption of memory technologies and the need for low power computers meant that phase change memories utilizing lower amount of power for operation, in combination with other attributes such as high capacity and durability of storage may prompt an industry wide switch in memory technologies, first in higher end laptops and mobile devices, and subsequently, with cost optimization, provision of the memory technology in lower end devices.

Conflicts of interest: The author declares no conflicts of interest.

Funding: No funding was used in this work.

\section{References}

1 Salinga, M. et al. Monatomic phase change memory. Nature Materials 17, 681-685, doi:10.1038/s41563-018-0110-9 (2018).

2 Burr, G. W. et al. Recent Progress in Phase-Change Memory Technology. IEEE Journal on Emerging and Selected Topics in Circuits and Systems 6, 146-162, doi:10.1109/JETCAS.2016.2547718 (2016).

3 Xiong, F. et al. in 2016 IEEE International Electron Devices Meeting (IEDM). 4.1.1-4.1.4.

4 Jones, R. O. Phase change memory materials: Rationalizing the dominance of $\mathrm{Ge} / \mathrm{Sb} / \mathrm{Te}$ alloys. Physical Review B 101, 024103, doi:10.1103/PhysRevB.101.024103 (2020).

5 Sarwat, S. G. Materials science and engineering of phase change random access memory. Materials Science and Technology 33, 1890-1906, doi:10.1080/02670836.2017.1341723 (2017).

6 Zhang, W., Mazzarello, R., Wuttig, M. \& Ma, E. Designing crystallization in phase-change materials for universal memory and neuro-inspired computing. Nature Reviews Materials 4, 150-168, doi:10.1038/s41578-018-0076-x (2019).

7 Lotnyk, A., Behrens, M. \& Rauschenbach, B. Phase change thin films for non-volatile memory applications. Nanoscale Advances 1, 3836-3857, doi:10.1039/C9NA00366E (2019).

8 Le Gallo, M. \& Sebastian, A. An overview of phase-change memory device physics. Journal of Physics D: Applied Physics 53, 213002, doi:10.1088/1361-6463/ab7794 (2020).

9 Navarro, G. et al. in 2018 IEEE International Memory Workshop (IMW). 1-4. 